\title{
刚塑性可压缩材料的变分原理 ${ }^{*}$
}

\author{
刘 相 华 \\ (东北工学院加工系, 沈阳)
}

\section{关调刚塑性有限元、可压缩法、变分原理}

自 1979 年森谦一郎等提出刚塑性有限元中的可压缩法 ${ }^{[1]}$ 以来, 已在分析金属成形过程中 得到了广泛的应用. 刚塑性有限元的理论基础之一是刚塑性材料的第一变分原理, 在满足不 可压缩条件下的这个变分原理已得到证明 ${ }^{[2]}$, 但对于㞌服条件与静水压力有关的刚塑性可压 缩材料 ${ }^{[3]}$, 类似的变分原理能否成立是需要加以证明的, 这个问题尚未得到解决.

\section{一、刚整性可压缩材料的基本方程}

1. 屈眼杂件 假设屈服与静水压力有关:

$$
\begin{aligned}
\sigma= & {\left[\frac { 1 } { 2 } \left(\left(\sigma_{x}-\sigma_{y}\right)^{2}+\left(\sigma_{y}-\sigma_{x}\right)^{2}+\left(\sigma_{z}-\sigma_{x}\right)^{2}\right.\right.} \\
& \left.\left.+6\left(\tau_{x y}^{2}+\tau_{y z}^{2}+\tau_{x x}^{2}\right)\right)+g \sigma_{m}^{2}\right]^{3},
\end{aligned}
$$

这里 $\sigma_{m}$ 为静水压力, $g$ 是与材料的可压缩程度有关的小正值常量.（1）式所表示的屈服条件 为 Mises 屈服圆柱内的旋转椭球体, $g$ 值越小,越接近 Mises 屈服条件,如图 1 所示.

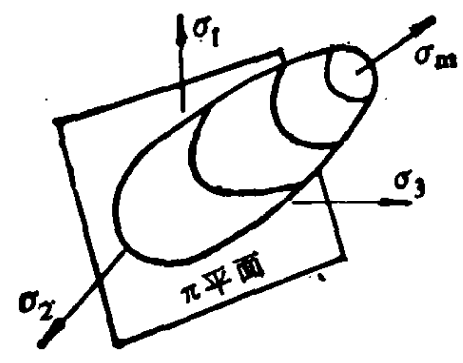

8

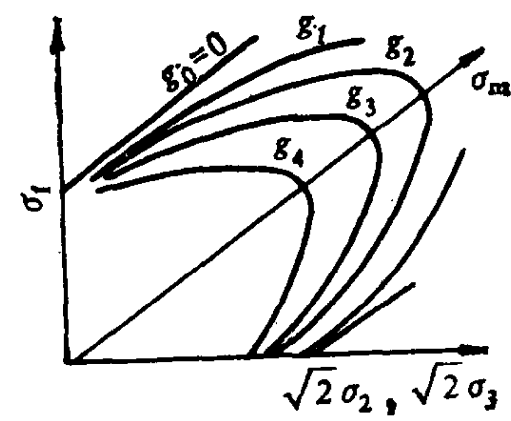

b

图 1 刚望性可压缩材料屈服函数的几何形状

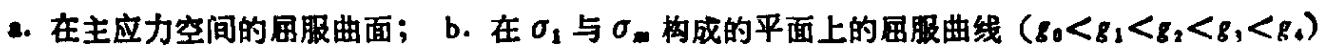

2. 等效形遗度设屈服函数为塑性势, 利用(1)式可导出刚塑性可压缩材料等效变形 速度的表达式

$$
\dot{\varepsilon}=\left[\frac { 2 } { 9 } \left(\left(\dot{\varepsilon}_{x}-\dot{\varepsilon}_{y}\right)^{2}+\left(\dot{\varepsilon}_{y}-\dot{\varepsilon}_{x}\right)^{2}+\left(\dot{\varepsilon}_{x}-\dot{\varepsilon}_{x}\right)^{2}\right.\right.
$$

- 滀家白结科学基金算助项目. 


$$
\left.\left.+\frac{3}{2}\left(\dot{\gamma}_{x y}^{2}+\dot{\gamma}_{y y}^{2}+\dot{\gamma}_{x x}^{2}\right)\right)+\frac{1}{g} \dot{\varepsilon}_{y}^{2}\right]^{\frac{1}{2}},
$$

这里 8 , 为体积变形速度.

\section{3. 应力-变形速度关系}

$$
\sigma_{i j}=\frac{\vec{\sigma}}{\dot{\bar{\varepsilon}}}\left[\frac{2}{3} \dot{\varepsilon}_{i j}+\delta_{i j}\left(\frac{1}{g}-\frac{2}{9}\right) \cdot \varepsilon_{i}\right],
$$

这里 $\delta_{i j}$ 为 $\mathrm{Kronecker}$ 符号, $\dot{\varepsilon}_{i j}$ 为变形速度张量.

$$
\dot{\varepsilon}_{i j}=-\frac{1}{2}\left(u_{i, j}+u_{i, i}\right) \text {. }
$$

用刚塑性有限元中的可压缩法分析金属成形过程时,可以利用(3)式从变形速度场中直接 得出应力场, 而不必引人 Lagrange 乘子, 这就使末知数的数目大为减少, 加快了计算速度.

\section{二、刚塑性可压缩材料的变分原理}

在满足 (1)、(4)式和边界速度已知条件的一切动可容速度场中, 便以下泛函取得最小值的 必为本问题的正确解:

$$
\phi=\int_{V} \sigma \dot{\varepsilon} d V-\int_{s_{p}} \bar{p}_{i} u_{i} d S \text {. }
$$

证 为推导方便,我们引人下面二个向量:

$$
\begin{aligned}
\left\{\sigma_{2}\right\}^{T}= & \left\{\frac{1}{\sqrt{2}}\left(\sigma_{x}-\sigma_{y}\right) \frac{1}{\sqrt{2}}\left(\sigma_{y}-\sigma_{x}\right) \frac{1}{\sqrt{2}}\left(\sigma_{x}-\sigma_{x}\right)\right. \\
& \left.\sqrt{3} \tau_{x y}, \sqrt{3} \tau_{y}, \sqrt{3} \tau_{x x} \sqrt{g} \sigma_{m}\right\}, \\
\left\{\dot{\varepsilon}_{x}\right\}^{T}= & \left\{\sqrt{\frac{2}{9}}\left(\dot{\varepsilon}_{x}-\dot{\varepsilon}_{y}\right) \sqrt{\frac{2}{9}}\left(\dot{\varepsilon}_{y}-\dot{\varepsilon}_{x}\right) \sqrt{\frac{2}{9}}\left(\dot{\varepsilon}_{x}-\dot{\varepsilon}_{x}\right)\right. \\
& \left.\frac{1}{\sqrt{3}} \dot{\gamma}_{x y} \frac{1}{\sqrt{3}} \dot{\gamma}_{y z} \frac{1}{\sqrt{3}} \dot{\gamma}_{x x} \frac{1}{\sqrt{g}} \dot{\varepsilon}_{v}\right\} .
\end{aligned}
$$

等效应力与等效变形速度可表示为

$$
\begin{aligned}
& \vec{\sigma}^{2}-\left\{\sigma_{2}\right\}^{T}\left\{\sigma_{2}\right\}, \\
& \delta^{2}=\left\{\delta_{2}\right\}^{T}\left\{\varepsilon_{2}\right\} .
\end{aligned}
$$

以下用上角标有 $*$ 的表示动可容值,不标 $*$ 为正确值,利用 Schwarz 不等式得到

$$
\left\{\sigma_{2}\right\}^{T}\left\{\varepsilon_{h}^{*}\right\} \leqslant \sigma^{*} \boldsymbol{\varepsilon}^{*} \text {. }
$$

容易验证

两式相减可得

$$
\left\{\sigma_{\lambda}\right\}^{T}\left\{\dot{\varepsilon}_{2}\right\}-\sigma \cdot \dot{\varepsilon}-\sigma_{i} \dot{\varepsilon}_{i}
$$

$$
\sigma\left(\xi^{*}-\dot{\varepsilon}\right) \geqslant\left\{\sigma_{2}\right\}^{T}\left(\left\{\dot{\varepsilon}_{1}^{*}\right\}-\left\{\dot{\varepsilon}_{2}\right\}\right),
$$

或

$$
\sigma\left(\dot{\varepsilon}^{*}-\dot{\varepsilon}\right) \geqslant \sigma_{i j}\left(\dot{\varepsilon}_{i}^{*}-\dot{\varepsilon}_{i j}\right),
$$

将(12)式写成积分形式

$$
\int_{\nu} \sigma \dot{\varepsilon}^{*} d V-\int_{\nu} \sigma_{i j} \varepsilon_{i j}^{*} d V \geqslant \int_{\nu} \sigma \varepsilon d V-\int_{V} \sigma_{i j \xi_{i j} d V}
$$


利用(4)式和力平衡微分方程、外力边界条件和速度边界条件, 并利用格林公式可以导出

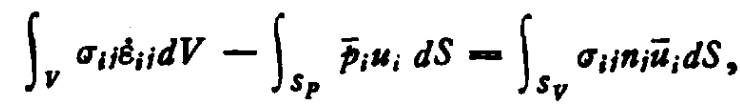

$$
\begin{aligned}
& \int_{V} \sigma_{i j} \dot{\varepsilon}_{i j}^{*} d V-\int_{S_{p}} \bar{p}_{i} u_{i}^{*} d S-\int_{s_{\nu}} \sigma_{i j} n_{j} \bar{u}_{i} d S .
\end{aligned}
$$

将(14)、(15)式代人(13)式得

$$
\begin{gathered}
\int_{V} \vec{\sigma} \varepsilon^{*} d V-\int_{S_{p}} \bar{p}_{i} u_{i}^{*} d S \geqslant \int_{V} \dot{\varepsilon} \sigma d V-\int_{s_{p}} \bar{p}_{i} u_{i} d S . \\
\text { 三、应用及实验验证 }
\end{gathered}
$$

应用上述刚塑性可压缩材料的变分原理,通过把可压缩参数 $g$ 取得很小 $(g \leqslant 0.01)$ 的办 法, 可用有限元法来求解金属成形问题. 作者利用这种方法求解了万能孔型中轧制 $\mathrm{H}$ 型钢的 问题 ${ }^{[10}$, 并在水平辊直径为 $\phi 325 \mathrm{~mm}$. 立辊直径为 $\phi 212.5 \mathrm{~mm}$. 轧制速度为 $0.22 \mathrm{~m} / \mathrm{s}$ 的实验 轧机上轧铅件进行了实验验证. 图 2 和图 3 给出了平均单位压力和轧制力矩的有限元计算值 与实测值的比较, 由此可见计算值与实测值符合良好. 这表明以刚塑性可压缩材料变分原理 为基础用有限元法求解金属成形过程是行之有效的.

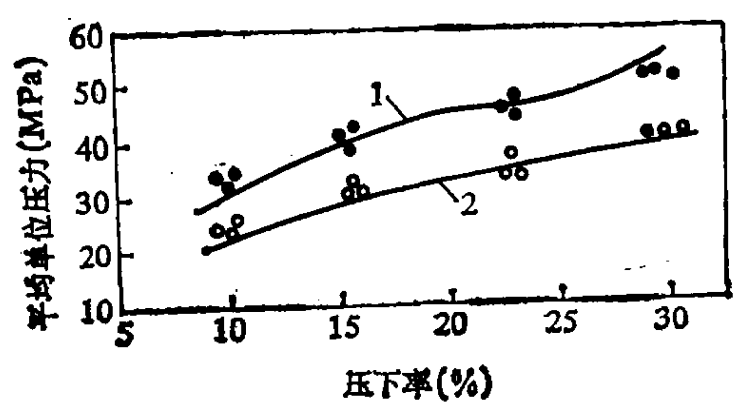

国 2 平均单位压力的计算值与实测值的比较 1.运部计勧值；2.边部计算值. - 需部实湖借; 0 边部实测值

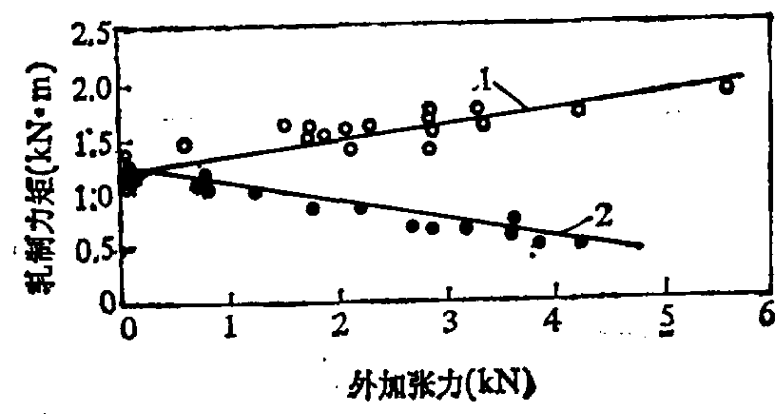

图 3 轧制力矩的计算值与实测值的比较 1.后张力计算值；2.前张力计算住. 0 后张力实测值; ・前张力实测值

\section{四、结论}

刚塑性可压缩材料与理想刚塑性材料相比,放松了体积不变约束条件,此时第一变分原理 吟然成立. 而用刚塑性可压缩材料模型可使有限元的求解过程简化, 减少未知数个数, 从变形 速度场中直接求出应力场. 这种材料模型不仅适用于粉末烧结材料, 也可通过把可压缩参数 $\boldsymbol{g}$ 取得很小来处理金属材料的塑性成形问题，此时体积不变条件是近似地满足的。

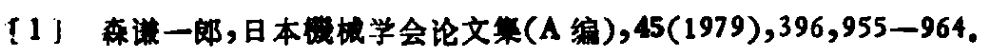

[2] 钱伟长,变分法及有限元(上册), 科学出版社, 1980,588.

[3] Shims, S., Int. J. Mech. Sci., 18(1976), 285.

(4) 刘相华、白光洞,力学与实战, 北京大学出版社, 1988, 81 . 